\title{
Detección molecular del virus de la hepatitis E en hígados de cerdo destinados al consumo humano en el estado de Nuevo León, México
}

Marco Antonio Cantú-Martínez, M en C,(1) Artur Xavier Roig-Sagués, D en C,(2) Sibilina Cedillo-Rosales, D en C,(I) Diana Elisa Zamora-Ávila, D en C,(I) Ramiro Ávalos-Ramírez, D en C.(I)

\author{
Cantú-Martínez MA, Roig-Sagués AX, Cedillo-Rosales S, \\ Zamora-Ávila DE, Ávalos-Ramírez R. \\ Detección molecular del virus de la hepatitis $\mathrm{E}$ en \\ hígados de cerdo destinados al consumo \\ humano en el estado de Nuevo León, México. \\ Salud Publica Mex 20I3; 55: 193-195.
}

\begin{abstract}
Resumen
Objetivo. Detección molecular del virus de la hepatitis $\mathrm{E}$ (VHE) en hígado de cerdo para consumo humano en Nuevo León, México. Material y métodos. Se analizaron 127 hígados de cerdo (87 obtenidos de rastros TIF, y 40 de carnicerías) mediante RT-PCR semianidado para amplificar un fragmento de $212 \mathrm{pb}$ del gen ORF2 del VHE. Resultados. El 19.5\% (I7) de los hígados de rastros y 22.5\% (9) de carnicerías fueron positivos. La secuenciación mostró $94-95 \%$ de homología con el genotipo 3. Conclusiones. Los resultados indican que el VHE circula en granjas porcinas del estado, lo que constituye una probable fuente de contaminación para los productos cárnicos porcinos.
\end{abstract}

Palabras clave: hepatitis E; VHE; hígados de cerdo; México
Cantú-Martínez MA, Roig-Sagués AX, Cedillo-Rosales S, Zamora-Ávila DE, Ávalos-Ramírez R.

Molecular detection of hepatitis $E$ virus in

pig livers destined for human consumption

in the state of Nuevo Leon, Mexico.

Salud Publica Mex 2013; 55: 193-195.

\begin{abstract}
Objective. Molecular detection of $\mathrm{HEV}$ in pig livers destined for human consumption in Nuevo Leon, Mexico. Materials and methods. 87 livers were collected from pigs slaughtered in TIF and 40 livers from butchers. A $212 \mathrm{pb}$ fragment of HEV ORF2 gene was amplified by semi-nested RT-PCR. Results. $19.54 \%$ (I7) of tif's and 22.5\% (9) of buthcer's livers were positive for HEV. Sequencing of the amplified products showed a $94 \%-95 \%$ homology with the sequences reported for genotype 3. Conclusions. Our results indicate that HEV is circulating in swine herds in the state, constituting a probable source of contamination of pig meat products.
\end{abstract}

Keywords: hepatitis E; HEV; pork livers; Mexico

(I) Departamento de Virología, Facultad de Medicina Veterinaria y Zootecnia, Universidad Autónoma de Nuevo León. Nuevo León, México.

(2) Fac. de Veterinaria, Departament de Ciència Animal I dels Aliments, Universidad Autónoma de Barcelona. Barcelona, España.

Fecha de recibido: 25 de junio de 2012 - Fecha de aceptado: 6 de diciembre de 2012 Autor de correspondencia: Dr. Ramiro Ávalos Ramírez. Departamento de Virología, Facultad de Medicina Veterinaria y Zootecnia, Universidad Autónoma de Nuevo León. Av. Francisco Villa s/n, col. Ex Hacienda el Canadá Escobedo. 66050 Nuevo León, México. Correo electrónico: raavara@hotmail.com 
$E^{1}$ virus de la hepatitis E (VHE) es uno de los cinco virus hepatotropos que afectan a humanos y se ha reconocido recientemente en varios países. El VHE causa la hepatitis $\mathrm{E}$, la cual se considera una zoonosis emergente que se transmite esencialmente mediante la ruta fecal-oral y su presentación clínica va desde la forma asintomática hasta la falla hepática fulminante (FHF). ${ }^{1}$ A nivel mundial, se han identificado cuatro genotipos del VHE. Los genotipos 1 y 2 se han encontrado exclusivamente en humanos y los genotipos 3 y 4 se han identificado en cerdos y otros animales en distintos países. ${ }^{2}$

Para el genotipo 3, encontrado en humanos y animales domésticos en zonas consideradas no endémicas, se considera al cerdo como el principal reservorio en la naturaleza y fuente primaria directa o indirecta de la infección hacia el humano. ${ }^{3}$ En 1997, Meng y colaboradores identificaron una nueva cepa porcina genética y serológicamente relacionada con cepas humanas en los Estados Unidos, ${ }^{4}$ lo que sugiere la posibilidad de infecciones humanas con cepas porcinas. En cifras oficiales en Nuevo León, en el periodo 2000 a 2007, se reportaron hasta $50 \%$ de casos de hepatitis en humanos por causas desconocidas. ${ }^{5}$ Debido a que nuestro país no cuenta con pruebas de diagnóstico serológico para el VHE, no se puede asegurar si dentro de estos casos se encuentre involucrado este virus. El objetivo de este trabajo es detectar mediante RT-PCR semianidado el VHE en muestras de hígados de cerdos destinados al consumo humano en el estado de Nuevo León, México.

\section{Material y métodos}

Durante 2010 y 2011 se recolectaron un total de 87 hígados de cerdos de un rastro tipo TIF de Nuevo León. Los animales procedían de 24 granjas porcinas de 11 municipios conurbanos al área metropolitana de Monterrey. Además, se incluyeron 40 hígados de centros de venta de 10 diferentes municipios de la misma área. Se obtuvo el ARN total de cada una de las muestras por el método de TRIzol Reagent. La síntesis del ADN complementario y RT-PCR semianidado se realizó utilizando un estuche comercial (Superscript IIl One-Step) para amplificar un fragmento de 212 pb del gen ORF 2 del VHE, utilizando los iniciadores recomendados por Martin y colaboradores. ${ }^{6}$ La amplificación se realizó en un termociclador con 35 ciclos (desnaturalización $94^{\circ} \mathrm{C} 50$ s, alineación $48^{\circ} \mathrm{C}$ 30s y amplificación a $72^{\circ} \mathrm{C} 60 \mathrm{~s}$ ) con incubación final a $72^{\circ} \mathrm{C}$ durante 7 minutos. ${ }^{6}$ Los productos de amplificación se analizaron por electroforesis en gel de agarosa al $1.5 \%$ y se documentaron previa visualizaron bajo la luz UV del transiluminador. Las amplificaciones de 15 muestras positivas se purificaron a partir de los geles de agarosa utilizando el estuche "Purelink Quik gel Extracción", acorde a las instrucciones del fabricante. Posteriormente, dos de los productos amplificados se secuenciaron en un ABI PRISM 310 Genetic Analyzer AB utilizando el estuche ABI PRISM bigDye Terminator v3.1 Secuencing kit y se analizaron con el software DNAstar lasergene 10. Las secuencias resultantes se analizaron para buscar homología con las secuencias reportadas del VHE en el GenBank. El protocolo científico y ético del estudio fue aprobado por la subdirección de posgrado de la Facultad de Salud Publica y Nutrición de la Universidad Autónoma de Nuevo León.

\section{Resultados}

De las 87 muestras analizadas, 17 (19.5\%) fueron positivas a la amplificación para VHE. Estas muestras provenían de 11 granjas distintas (figura $1 \mathrm{a}, \mathrm{b}$ ). Una las muestras positivas, confirmada mediante secuenciación, fue utilizada como control positivo para los ensayos posteriores. De los 40 hígados de centros de venta de carne, $9(22.5 \%)$ fueron positivos a VHE. Estos provenían de cuatro municipios del estado: Salinas Victoria, Cerralvo, Cadereyta Jiménez y Montemorelos (figura 2 a, b). Las secuencias detectadas pertenecen al genotipo 3 y muestran $94 \%$ de homología con la reportada en cerdos por Huang FF y colaboradores en EUA en 2002 $(\mathrm{AF} 466664.1)^{7}$ y con la reportada por Takahashi M. y

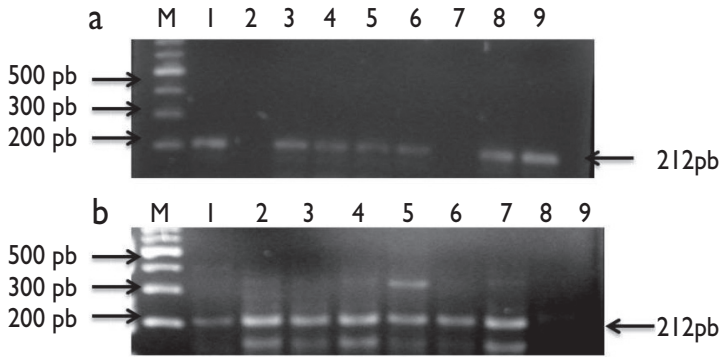

Figura I A Y B. Amplificación de Un fRAgmento del gen ORF de VHE de hígAdos PROVENIENTES de RASTROS. A) Electroforesis en GEL de AGAROSA AL I.5 \% TEÑIDO CON BROMURO DE ETIDIO. M: MARCADOR DE PESO MOLECULAR I00 PB, CARRIL I: CONTROL POSITIVO, CARRIL 2: CONTROL Negativo, CARriles 3-9: MUestras de hígados. B) M: MARCADOR DE PESO MOLECULAR I00PB, CARRIL I: CONTROL POSITIVO, CARRILES 2-7: MUESTRAS DE HÍGADOS, CARRIL 8: CONTROL NEGATIVO 
$\begin{array}{llllllllll} & M & 2 & 3 & 4 & 5 & 6 & 7 & 8 & 9\end{array}$
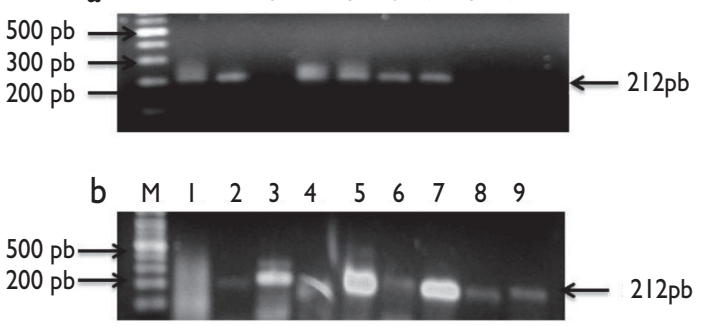

Figura 2 a y b. Amplificación de Un fRAgmento del gen ORF DE VHE dE hígados PROVENIENTES DE CARNICERÍAS. A) Electroforesis en Gel de agarosa al I.5 \% teÑIdo CON BROMURO DE ETIDIO. M: MARCADOR DE PESO MOLECULAR I O0PB, CARRILES I Y 2: CONTROLES POSITIVOS, CARRIL 3: CONTROL NEGATIVO, CARRILES 4-9 MUESTRAS DE HígAdos. B) M: Marcador de Peso molecular I00PB, CARril I: CONTROL NEGATIVO, CARRIL 2: CONTROL POSITIVO, CARRILES 3-9 MUESTRAS DE HÍGADOS

colaboradores en 2003 (AB094209.1) para cepas porcinas. Es interesante resaltar que una de las secuencias detectadas mostró una homología mayor a 95\% con una cepa del VHE reportada en 2008 en pacientes en Japón (AB360348.1). 2,8

\section{Conclusión}

Actualmente se ha demostrado que los porcicultores $\mathrm{y}$ veterinarios que manejan cerdos tienen un mayor riesgo de infectarse con el $\mathrm{VHE},{ }^{9,10}$ y además, existen informes que asocian el consumo de hígado de cerdo o carne cruda con el desarrollo de hepatitis E. ${ }^{11,12}$ Nuestros resultados sugieren que existe la posibilidad de que los pacientes catalogados en nuestro país con hepatitis por causas desconocidas presenten una infección por VHE no reportada tal vez por falta de pruebas de diagnóstico específicas. Por otro lado, en EUA se ha reportado una secuencia parcial de una variante en humanos del VHE (VHE-US1) aislada de pacientes que sufrieron hepatitis viral aguda, 13,14 generando evidencia de la presencia de nuevas cepas del virus en América del Norte diferente a las secuencias reportadas previamente para las cepas de EUA, Birmania y México. No se conoce el origen de estas nuevas cepas, sin embargo, existe la hipótesis de que sean de origen animal y que deberán existir varios reservorios potenciales del VHE.

Este es el primer estudio que aporta evidencia de la circulación del genotipo 3 de VHE en granjas porcinas, cerdos sacrificados en rastro y carnicerías de Nuevo
León. La presencia del virus puede constituir un factor de riesgo importante para la salud humana.

Declaración de conflicto de intereses: Los autores declararon no tener conflicto de intereses.

\section{Referencias}

I. Lu L, Li C, Hagedorn CH. Phylogenetic analysis of global hepatitis $\mathrm{E}$ virus sequences: genetic diversity, subtypes and zoonosis. Rev Med Virol 2006; 16:5-36. PMID: I6I75650.

2. Takahashi M, Nishizawa T, Miyajima H, Gotanda Y, lita T, Tsuda F, et al. Swine hepatitis $E$ virus strains in Japan form four phylogenetic clusters comparable with those of Japanese isolates of human hepatitis $E$ virus. J Gen Virol 2003;84:85I-862. PMID:I2655086.

3. Halbur PG, Kasorndorkbua C, Gilbert C, Guenette D, Potters MB, Purcell RH, et al. Comparative pathogenesis of infection of pigs with hepatitis $E$ viruses recovered from a pig and a human. J Clin Microbiol 200।;39: 918-923. PMID: II 230404.

4. Meng X, Purcell J, Halbur RH, Lehman PG, Webb JR, Tsareva DM, et al. A novel virus in swine is closely related to the human hepatitis $E$ virus. Proc Natl Acad Sci USA 1997;94:9860-9865. PMID:92752I6.

5. Sistema Único de Información para la Vigilancia Epidemiológica/Dirección General de Epidemiología/SSA 1997. México: SUIVE/SSA, 2007.

6. Martin M, Segale's J, Huang FF, Guenette DK, Mateu E, de Deus N, et al. Association of hepatitis $\mathrm{E}$ virus (HVE) and postweaning multisystemic wasting syndrome (PMWS) with lesions of hepatitis in pigs. Veterinary Microbiology 2007; I22:I6-24 PMID:I7270366.

7. Huang FF, Haqshenas G, Guenette DK, Halbur PG, Schommer SK, Pierson FW, et al. Detection by reverse transcription-PCR and genetic characterization of field isolates of swine hepatitis $E$ virus from pigs in different geographic regions of the United States. J Clin Microbiol 2002;40: I326-I332. PMID: I I 923352

8. Takahashi M, Hoshino Y, Tanaka T, Takahashi H, Nishizawa T, Okamoto $\mathrm{H}$. Production of monoclonal antibodies against hepatitis $\mathrm{E}$ virus capsid protein and evaluation of their neutralizing activity in a cell culture system. Arch Virol 2008;I53(4):657-666 PMID:I8266052.

9. Drobeniuc J, Favorov MO, Shapiro CN, Bell BP, Mast EE, Dadu A, et al. Hepatitis E virus antibody prevalence among persons who work with swine. J Infect Dis 200I;184:1594-1597.

10. Meng XJ, Wiseman B, Elvinger F, Guenette DK, Toth TE, Engle RE, et al. Prevalence of antibodies to hepatitis $E$ virus in veterinarians working with swine and in normal blood donors in the United States and other countries. J Clin Microbiol 200 I;40: I I7-122. PMID: I I773I03.

I I. Yazaki Y, Mizuo H, Takahashi M, Nishizawa T, Sasaki N, Gotanda Y, et al. Sporadic acute or fulminant hepatitis $E$ in Hokkaido, Japan, may be food-borne, as suggested by the presence of hepatitis $E$ virus in pig liver as food. J Gen Virol 2003; 84:235I-2357. PMID:I29I7455.

12. Tei S, Kitajima N, Takahashi K, Mishiro S. Zoonotic transmission of hepatitis $E$ virus from deer to human beings. Lancet 2003;362:37I-373. PMID:I29070II.

I3. Hsieh SY, Meng XJ, Wu YH, Liu ST, Tam AW, Lin DY, et al. Identity of a novel swine hepatitis $E$ virus in Taiwan forming a monophyletic group with Taiwan isolates of human hepatitis E virus. J Clin Microbiol 1999;37:3828-3834. PMID: I0565892.

14. Kabrane-Lazizi Y, Zhang M, Purcell RH, Miller KD, Davey RT, Emerson SU. Acute hepatitis caused by a novel strain of hepatitis $E$ virus most closely related to United States strains. J Gen Virol 200I;82:I687-I693. PMID: 10463689 